\title{
Prophylactic Fixation of Impending Fractures
}

\author{
Bahaa Kornah $^{1,2^{*}}$, Hesham Safwat ${ }^{1,2}$, Tharwat Abdel Ghany ${ }^{1,2}$ and Mohamed Abdel-AAl ${ }^{1,2}$ \\ ${ }^{1}$ Al-Azhar University, Cairo, Egypt \\ ${ }^{2}$ Manshet El bakey Hospital, Alazhar University, Cairo, Egypt
}

*Corresponding author: Bahaa Kornah, MD, Consultant Orthopedic Surgeon, Al-Azhar University, Cairo, Egypt, 90 D Ahmed Orabi St. Mohandessin, Giza, Egypt, Tel: 00201223433418; Fax: 00202 33032557; E-mail: bkornah@yahoo.co.uk

Received date: Jul 07, 2016; Accepted date: Aug 04, 2016; Published date: Aug 11, 2016

Copyright: (c) 2016 Kornah B, et al. This is an open-access article distributed under the terms of the Creative Commons Attribution License, which permits unrestricted use, distribution, and reproduction in any medium, provided the original author and source are credited.

\begin{abstract}
Aim: Pathologic fractures occur as a result of weakening of the mechanical properties of bone. There are many conditions, which lead to bone softening. There are neoplastic and non-neoplastic diseases that cause pathologic fractures. The aim is to evaluate and to highlight on value of prophylactic fixation of impending fractures in abnormal bone situation to prevent occurrence of pathological fracture and its complications.

Patients and methods: Between 2003 and 2009, study on forty-nine patient (35 females and 14 males) between the age of 20 and 65 years with an average age of 49.9 years with expected possibility of fractures of lower extremities. The entire patient with fractures risks prophylactic fixation has done. Different types of fixation either surface plating (dynamic hip screws), medullary (Gamma nails or locking nail) or external fixation in cases unsuitable for surgery was used according to the type and the site of the deformity in combination of management of primary condition.
\end{abstract}

Results: Most patients had significant relief of pain. $35(71.5 \%)$ of patients with impending fracture were ambulatory after therapy and able to walk outdoor while $10(20.5 \%)$ of patients could walk inside door. Three patients $(6 \%)$ using wheel chair, and they were on renal dialysis. Only one patient $(2 \%)$ was not able to walk, and he was not cooperative for unknown reason. The mean duration of hospitalization was 21 days (range from 7 to 35 days). That time included preoperative period of investigation and preparation and post-operative surgery and rehabilitation.

Discussion: Pathological fractures create a serious morbidity in patients with bone disease. Orthopedic surgeons who look after patients with skeletal lesions should focus on proactive treatments designed to prevent pathologic fractures before they occur. Prophylactic fixations have decreased morbidity compared with patient's sustained fractures before fixation.

Conclusion: Surgical fixation of fractures in weight-bearing long bones with impending fractures provides pain relief, and a functionally stable and durable construct. It helps early an ambulation and prevents fracture's complication. It allows independent function and avoids irretrievable catastrophes.

Keywords: Fractures; Prophylactic fixation; Pathological fractures; Impending fractures

\section{Introduction}

Changes in the bone are the results from a continuous process of bone resorption and bone formation known as "bone remodeling" which involves bone growth, changes in bone density and calcium level regulation in the body. This process occurs throughout a life span. Once the bone mass has been achieved, bone formation generally equal to bone resorption and bone density remains unchanged. This is called peak bone density [1]. In normal bone, shear stresses are distributed evenly in cross section.

Pathological fractures occur as a result of an underlying process; the so-called bone softening disease; that weakens the mechanical properties of bone. There are neoplastic and non-neoplastic diseases that cause pathological fractures [2]. Bone softening is a condition which occurs almost only in adults, and is due to a loss of the lime-salts of the bone. The disease, which is comparatively rare, affect's women, principally. Many diseases that affect the metabolism typically result in bone softening because the osteoid, which is the bone matrix, does not calcify how it should, resulting in bones that bend, twist and fracture. Many medical conditions and chemical drug therapy can affect the bone strength, e.g. vitamin D deficiency and Phenytoin for treatment of epilepsy produce osteomalacia. The strength and integrity of bones depend on maintaining a delicate balance between bone resorption by osteoclasts and bone formation by osteoblasts [3].

Factors affect the biomechanics of bone and lead to pathological fractures are Bone defect size, Biological behavior of bone lesion, and the anatomic location of the lesion. Bone in these situations is subjected to what is called impending pathological fractures which definition is controversial. Diagnosis and estimation of fracture at risk with its decision for prophylactic fixation are difficult.

Pain; mostly, in the pelvis, is the first symptom with the bone softening disease. The pain could radiate towards the spinal column or thighs, and at first it is often thought to be rheumatic. Soon, however, 
Page 2 of 5

an uncertain dragging or peculiarly waddling gait becomes noticeable. Sitting causes pain and the bones bend. The spinal column is no longer able to carry the weight of the body. It curves backward and as a result; the patients grow shorter. When the disease has reached a more advanced stage, walking becomes impossible and the patients must remain in bed. The fractures typically are sustained after a low-energy mechanism with the presence of an existing characteristic stress fracture. However, it is unclear whether these patients are best treated conservatively or operatively.

Many authors recommend prophylactic osteosynthesis for all impending pathological fractures [4-7]. Many options are available to the orthopedic surgeon for reconstruction of long bones; this includes the usage of plates and screws, intramedullary nails, reconstruction nails, endoprosthesis and customized or massive endoprosthesis [2]. In literatures, little is mentioned about prophylactic fixation for nonneoplastic diseases that cause pathologic fractures.

\section{Patients and Methods}

\section{Patient's population}

Between 2003 and 2009, forty-nine patients (35 females (71.4\%) and $14(28.6 \%)$ males) with an average age of 49.9 years (20-65 years) and an expected lower extremities fractures were treated.

\begin{tabular}{|l|l|}
\hline Cause of bone softening & No. of patients \\
\hline Osteomalacia and osteoporosis & 25 \\
\hline Renal dialysis & 6 \\
\hline Secondary bone metastasis & 11 \\
\hline Rheumatoid arthritis & 1 \\
\hline Hyperparathyroidism & 2 \\
\hline Bone cyst & 3 \\
\hline Primary bone Malignant & 1 \\
\hline Total & 49 \\
\hline
\end{tabular}

Table 1: Clinical Data.

Pain was the most common symptom and was present in 35 patients (71.4\%). Other symptoms and signs were difficult to walk and limping. 25 patients $(51 \%)$ had looser zone and progressive decrease in neck shaft angle. Those patients were diagnosed as having osteomalacia. The remaining 24 patients (49\%) had different bone softening conditions (Table 1$)$. The femur was affected in 35 patients $(71 \%)$, and the tibia was affected in 14 patients (29\%) (Table 2).

\section{Patient's evaluation}

The diagnosis in all cases was done in a combined work between orthopedic, rheumatology, internal medicine and other necessary specialties. This was done by history, clinical, radiological and laboratory findings, in addition to biopsy.

Estimation of fracture at risk is a very important step to do prophylactic fixation. The fracture at risk is based on radiographic findings. The occurrence of pathological fractures depended upon many factors such as bone defect size, biological behavior of bone lesion, and the anatomic location.

\begin{tabular}{|c|c|c|c|c|}
\hline Site affected & $\begin{array}{l}\text { Number } \\
\text { patients }\end{array}$ & $\begin{array}{l}\text { Method of } \\
\text { fixation }\end{array}$ & Female & Male \\
\hline Femur & 12 & $\begin{array}{l}\text { Interlocking } \\
\text { femoral nail and } \\
\text { gamma nail }\end{array}$ & 5 & 7 \\
\hline Neck of femur & 20 & DHS & 13 & 7 \\
\hline Distal femur & 2 & $\begin{array}{l}\text { distal locked } \\
\text { femur plate }\end{array}$ & 1 & 1 \\
\hline Tibia & 12 & Interlocking nail & 6 & 6 \\
\hline Tibia & 2 & Locked plate & 1 & 1 \\
\hline Femur & 1 & External fixators & & 1 \\
\hline Total & 49 & & 26 & 23 \\
\hline
\end{tabular}

Table 2: Site Affected and Method of Management.

\section{Harington's criteria:}

$>50 \%$ destruction of diaphyseal cortices

$>50-75 \%$ destruction of metaphysis $(>2.5 \mathrm{~cm})$

Permeative destruction of the subtrochanteric femoral region

Persistent pain following irradiation

The size of pathological defects is classified as two types [8]:

1. Lesions less than the diameter of the involved bone and

2. Lesion that is greater than the diameter of bone on the maximum dimension.

Prophylactic fixation is recommended for lesions finer than $2.5 \mathrm{~cm}$ or finer than $50 \%$ of the cross-sectional bone destruction [9]. The use of imaging in treatment planning depends on whether the differential diagnosis includes primary benign or malignant lesions. In benign lesions, the diagnosis depends upon the age, radiological characteristics and location of the lesions and the presence or absence of progressive or multiple lesions. The natural history of some tumors may require more advanced imaging [10].

The traditional surgical treatment of benign bone tumors has been curettage and autologous bone graft or marginal resection of expendable bone lesions [10] and prophylactic fixation if doubt of segment stability. Biopsy was done for lesions to type of lesion exclude malignancy and then treats and fix prophylactic ally like the primary lesions.

Scoring system was proposed [11] in an attempt to identify the risk of sustaining a pathological fracture through a metastatic defect in long bone. This system is based on four characteristics: (1) site of lesion; (2) nature of lesion; (3) size of a lesion; and (4) pain. All the features were assigned in progressive scores ranging from 1 to 3 . According to Mirels' recommendation, prophylactic fixation is highly indicated for a lesion with an overall score of 9 or greater [11] (Table $3)$.

Mirels' score clinical recommendation is if $\leq 7$ then radiotherapy and observation, if 8 then use clinical judgment and $\geq 9$ then Prophylactic fixation. 
Excluded from this study is any patient who already developed pathological fracture. Different types of fixation either surface plating (dynamic hip screws) (Figures 1 and 2). Medullary (Gamma nails) (Figure 3) or external fixation in cases unsuitable for surgery (Figure 4) were used according to the type and the site of the deformity. In tibial cases, we used the tourniquet without blood evacuation in the limb.

\begin{tabular}{|l|l|l|l|}
\hline \multirow{2}{*}{ Variable } & \multicolumn{3}{|c|}{ Score } \\
\cline { 2 - 4 } & $\mathbf{1}$ & $\mathbf{2}$ & $\mathbf{3}$ \\
\hline Site & Upper limb & Lower limb & Peritrochanter \\
\hline Pain & Mild & Moderate & Functional \\
\hline Lesion & Basic & Mixed & Lytic \\
\hline Size & $<1 / 3$ & $1 / 3-2 / 3$ & $>2 / 3$ \\
\hline
\end{tabular}

Table 3: Mirel's scoring system (Mirels, 1989). It is based on four charecteristics: site of lesion, pain, nature of lesion and size of lesion. All the features were assigned progressive scores ranging from 1 to 3 .

All patients with medical conditions were first managed by the specialist to treat the primary causes and all patients were evaluated post operatively regarding to: discharge destination, mortality, morbidity, pain status and daily activity.

\section{Postoperative care}

The postoperative physical therapy largely depends on the type of construct used and the intra-operative observations made by the surgeon regarding the quality of bone, screw purchase, and overall stability of the construct. The goal is to achieve mobility and independence in order to improve the quality of life and to decrease cardiopulmonary complications that are associated with immobility in the elderly patient. Adequate pain control is necessary for participation in physical therapy.

DVT prophylaxis is very important in cancer patients that are immobilized. Bisphosphonates, radiation therapy, and chemotherapy should be used as indicated, keeping in mind that radiation and chemotherapy decrease wound healing and may be delayed.

\section{Results}

In this study, all patients with impending fracture lesions with an overall score of 9 or greater went for prophylactic fixation [11]. There were 35 female $(71.4 \%)$ and 14 male $(28.6 \%)$ between the age of twenty and 65 years with an average age of 49.9 years with bending fractures of lower extremities. The average duration of follow-up was 2.2 years (range, 6- 48 months). The average blood loss was $250 \mathrm{ml}$, and the average surgery time was 135 minutes (45 minutes to 180 minutes). Most of blood loss was in the femoral cases.

All patients began to stand up within the first week after surgery and ambulated partially from the second week. Two cases of hyperparathyroidism had a combined orthopedic and general surgical treatment. In this series of patients, there were no cases of wound infection, deep venous thrombosis or decubitus ulcer. Wound drainage was seen in two cases; one was hepatic patient with blood Coagulopathy and was fixed by external fixation. The discharge was sterile and was treated by plasma transfusion and control blood bleeding profile.
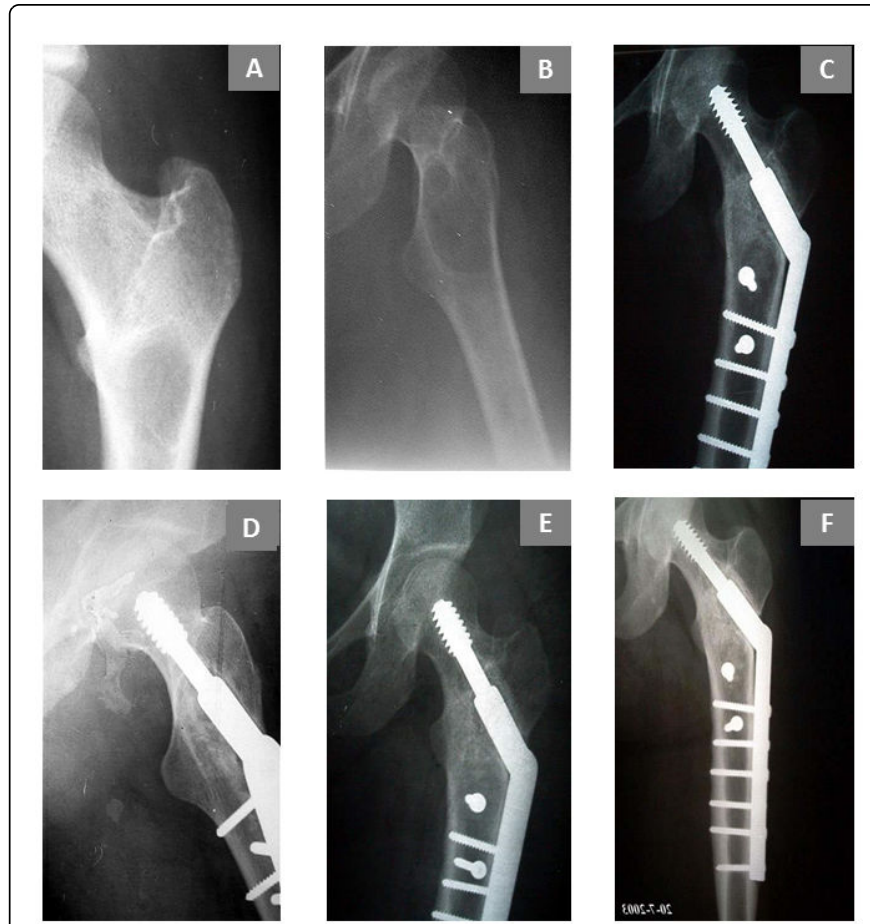

Figure 1: Preoperative $\mathrm{X}$-ray with osteolytic lesion at upper end femurs A and B. The lesions are greater than diameter of bone on maximum dimension and in high risk fractures. This defect of the femur will cause significant decrease in the mechanical performance of the femur when resisting external load and twisting. $\mathrm{C}$ and $\mathrm{D}$ show immediate postoperative prophylactic fixation by dynamic hip screws and bone grafting. E and $\mathrm{F}$ show follow up after 2 years with complete incorporation of the graft.

The second case was treated by antibiotic after debridement, the culture of which was negative and resolved during 2 months. There was no instrumentation failure, loosening or mortality.

Most patients had significant relief of pain. 35 (71.5\%) of patients with impending fracture were ambulatory after therapy and able to walk outdoor, while $10(20.5 \%)$ of patients were able to walk inside door. We had $3(6 \%)$ patients using wheel chair and they were on renal dialysis. Only one patient (2\%) was not able to walk and he was not cooperative for unknown reason (Figure 5).

\section{Discussion}

Pathological fractures create a serious morbidity in patients with bone disease. Orthopedic surgeons who treat patients with skeletal lesions should focus on proactive treatments designed to prevent pathological fractures before they occur. Prophylactic fixations have decreased morbidity compared with patient's sustained fractures before fixation [11]. The difficulty lies in determining patients requiring prophylactic fixation. Many different criteria have been suggested including, pain over the site of lesion, size and location of the lesion, whether the lesion is blastic or lytic or mixed, irradiation of the lesion and the type of primary tumor in metastatic lesions [12]. 


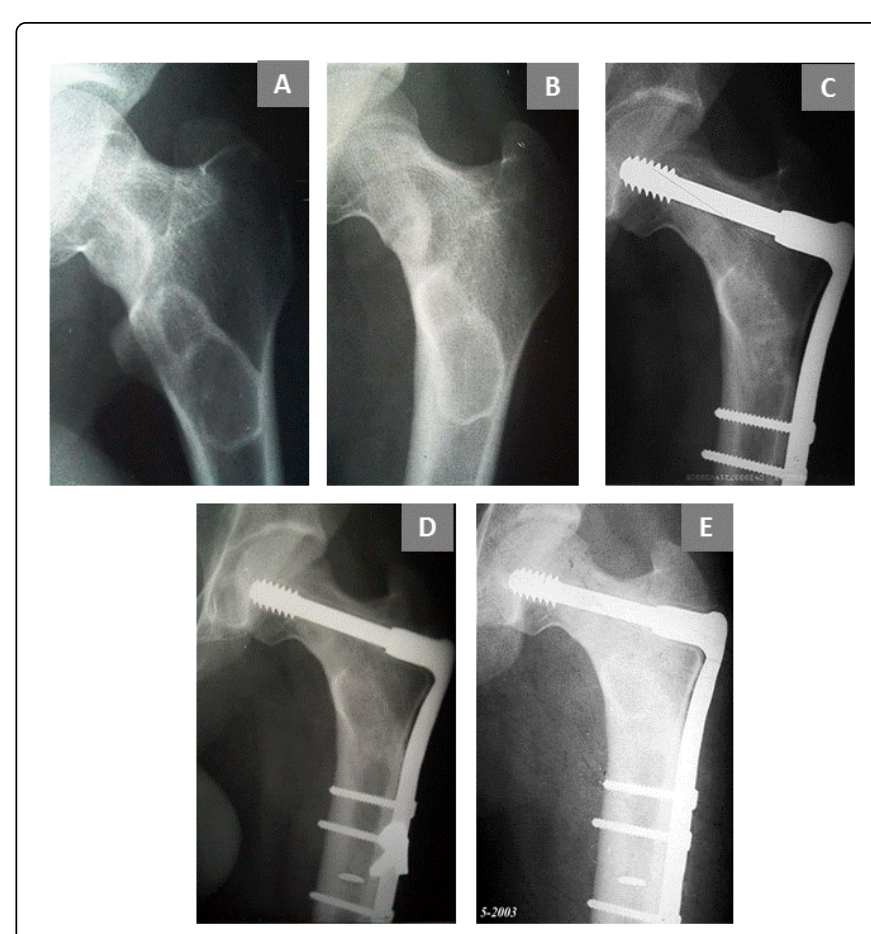

Figure 2: Preoperative X-rays of a lesion at the proximal end and neck of the femur (loser zone), the lesion is more than half of the diameter with an impending fracture at the neck of the femurs (A and $\mathrm{B})$. C and D post-operative prophylactic fractures fixation by dynamic condylar plate. E shows 2 years follow up.
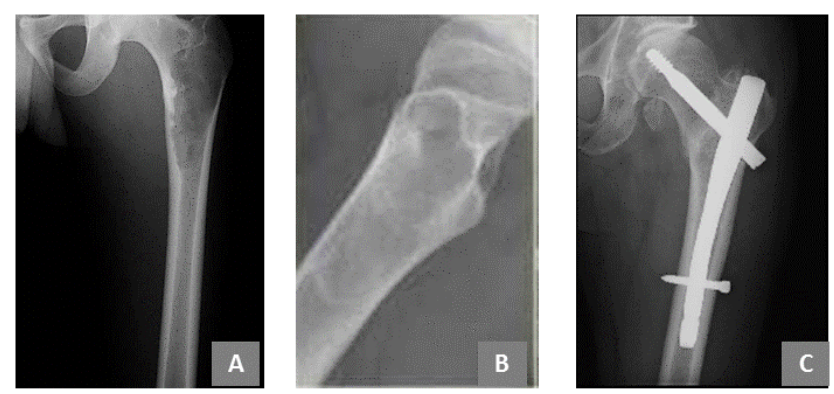

Figure 3: A and B Show an osteolytic lesion of the proximal end femur with high risk factors by increasing pain, the size of the lesion, radiographic appearance, localization, transverse/axial/ circumferential involvement of the cortex and the scoring system of Mirels. C Shows post-operative fixation after one year with mild resolution of the cyst and heterotrophic calcification at site of nail insertion and head of femur.

In order to determine which patient require prophylactic fixation to prevent pathological fracture, it is necessary to perform accurate and reliable risk evaluations. Many factors, including patient himself, nonneoplastic diseases that cause pathologic fractures, tumor nature (malignant or benign), pain and lastly, responsiveness to non-surgical treatment can affect the discussion [11]. The potential for pathological fracture can be assisted by size as the bone defect which could be classified under two types, these less than the diameter of the involved bone and those that are greater than the diameter of bone on the maximum dimension. Smaller lesions called stress riser while a large defects called open section defect [8].

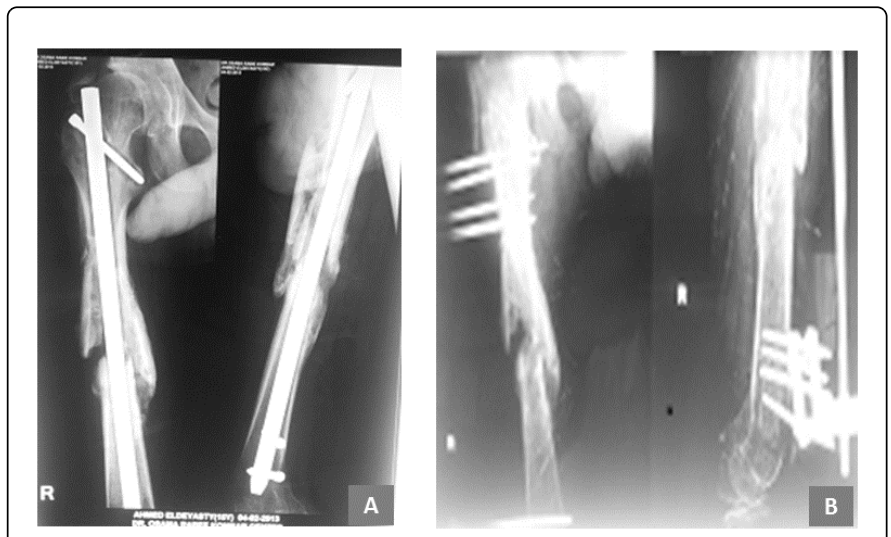

Figure 4: A) Shows an $x$-ray with post traumatic infected interlocking nail and partial healing of the fracture. B) After debridement of the infection, removal of the nail with the risk of sustaining a pathological fracture through a defect in long bone. A prophylactic external fixator was applied to avoid a pathological fracture.

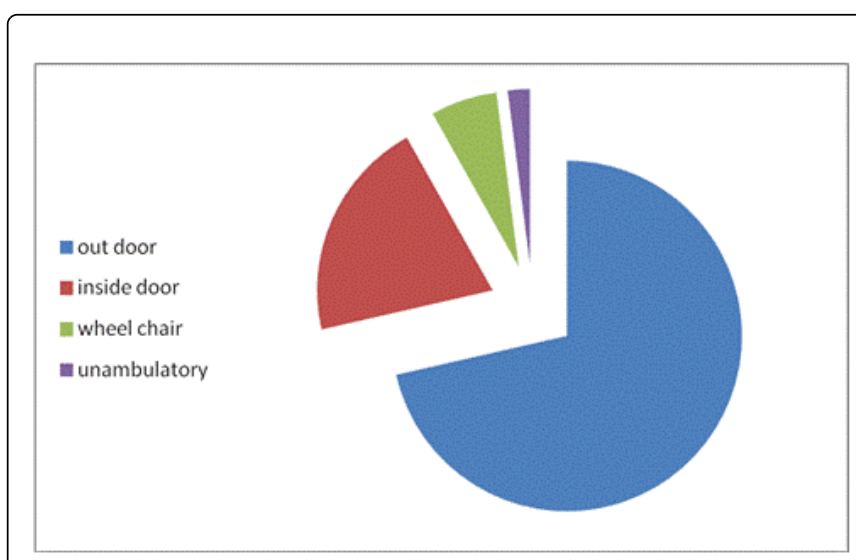

Figure 5: The mean duration of hospitalization was 21 days (range from 7 to 35 days). That time included preoperative period of investigation and preparation and post-operative surgery and rehabilitation.

It is of importance to stress on the anatomic location of the bony lesions for the assessment of potential fractures [13]. As the lower limbs to support body weight for ambulation, so, lesions in these areas are at increased risk for pathological fracture, specifically, those lesions in the proximal femur which are considered to be at the greatest risk for fracture among all long bone lesions [14].

The most important factor to be considered in deciding the treatment option for the management of impending bone fracture due to bone softening disease is the level of the patient's dysfunction and pain. Severe dysfunction or pain demands a treatment that predictably leads to a quick resumption of the painless activities of daily living and to prevent fractures. Prophylactic surgical treatment of impending fractures has been shown to improve outcomes [15]. Early 
management will help the patient to continue and shorten the treatment period and the need for assistance.

Prophylactic fixation has been recommended for lesions finer than $2.5 \mathrm{~cm}$ or finer than $50 \%$ of cross sectional bone destruction [9]. The consequences of pathological fracture in this area are significant so that prophylactic fixation prevents pathological complications [12,16].

Biologic behavior of bony lesions is another factor in determining the possibility for pathological fracture. Biologic aggressiveness can be inferred from whether the lesion is lytic or blastic or mixed (lytic and blastic). Zickel and Mouradian [17] reviewed 34 patients and found that lesions could be listed in ascending order of least likely to fracture are blastic lesions, mixed blastic and lytic lesion then purely lytic lesions, which are the most prone to pathological fracture.

The treatment of patients with an expected short life span as those suffering from metastatic disease is considered to be very important to help them. A bone that has lost its structural integrity, even though not grossly fractured, will not support weight bearing for months even if the metastasis is eliminated. Control of the metastatic tumor does not always equate with return to function [18].

It is a reasonable goal to avoid bone softening or bone metastasis to progress to pathological fracture. Function can almost always be returned to these patients with impending fractures. This can be done by surgical stabilization, which may be the best way to return the patient's function while he/she is being treated postoperatively with medical therapy, radiotherapy or chemotherapy.

Comprehensive management of patients with soft bone disease requires the participation of an orthopedic surgeon early in the clinical course. The orthopedic surgeon's role should be more than patching together fractured bones that have not responded to other treatment modalities. Early consultation and mutual follow-up will benefit a patient in maintaining independent function and avoid irretrievable catastrophes.

In this study, many devices were used. Most of the cases with hip affection due to osteomalacia were fixed by dynamic hip screws. These devices allow an open exposure of lesions' sites, which is useful in cases that require open biopsy and diagnostic confirmation. Intramedullary nails can be used for femoral shaft lesion if there is permeate destruction without significant focal size area of cortical loss. For most patients with impending fracture from femoral metastasis, insertion of reconstruction nail is favorable. This technique provides resistance to be torsional stresses as well as angular displacement throughout the full length of the femur, including the intertrochanteric and femoral neck areas [6].

External fixators were used in patients with medical conditions that prevent surgical interference. Active and prompt treatment of pathological fractures is justified. In the present series, all patients were relieved of pain immediately after fixation and as a consequence of the fracture treatment; it became easier to mobilize them. The length of the hospital stay is reduced and for those patients who cannot be mobilized because of their primary disease, the nursing care is made easier.

\section{Conclusion}

Surgical fixation of fractures in weight-bearing long bones with impending fractures provides pain relief, and a functionally stable and durable construct. It helps an early ambulation and prevents fracture complications. It allows independent function and avoids irretrievable catastrophes.

\section{References}

1. Hadjidakis DJ, Androulakis II (2006) Bone remodeling. Ann N Y Acad Sci 1092: 385-396.

2. Levine AM, Aboulafia AJ (2008) Pathologic Fractures in Browner: Skeletal Trauma (4th edn) W. B. Saunders Company, United States.

3. Rodan GA, Martin TJ (2000) Therapeutic Approaches to Bone Diseases. Science 289: 1508-1514.

4. Altman H (1953) Metallic fixation for pathologic fracture and impending fracture of long bones. J Int Coll Surg 19: 612-617.

5. Bremner RA, Jelliffe AM (1958) The management of pathological fracture of the major long bones from metastatic cancer. J Bone Joint Surg Br 40: 652-659.

6. Johnson EW (1957) Intramedullary fixation of pathological fractures. JAMA 163: 417-419.

7. Katzner M, Petit R, Schvingt E (1974) Surgical treatment of metastases and metastaic fractures of the long bones. Apropos of 53 palliative osteosynthesis. Rev Chir Orthop Reparatrice Appar Mot 60: 387-400.

8. Haentjens P, Casteleyn PP, Opdecam P (1993) Evaluation of impending fractures and indications for prophylactic fixation of metastases in long bones. Review of the literature. Acta Orthop Belg 59: 6-11.

9. Hipp JA, Springfield DS, Hayes WC (1995) Predicting pathologic fracture risk in the management of metastatic bone defects. Clin Orthop Relat Res 312: $120-135$.

10. Gibbs CP, Lewis VO, Peabody T (2005) Beyond bone grafting: techniques in the surgical management of benign bone tumors. Instr Course Lect 54: 497-503.

11. Mirels H (1989) Metastatic disease in long bones. A proposed scoring system for diagnosing impending pathologic fractures. Clin Orthop Relat Res 249: 256-264.

12. O'Donnell P (2013) Impending Fracture \& Prophylactic Fixation.

13. Harrington $\mathrm{KD}$ (1986) Impending pathologic fractures from metastatic malignancy: evaluation and management. Instr Course Lect 35: 357-381.

14. Keene JS, Sellinger DS, McBeath AA, Engber WD (1986) Metastatic breast cancer in the femur. A search for the lesion at risk of fracture. Clin Orthop Relat Res 203: 282-288.

15. Ward WG, Holsenbeck S, Dorey FJ, Spang J, Howe D (2003) Metastatic disease of the femur: surgical treatment. Clin Orthop Relat Res 415: S230S244.

16. Van Geffen E, Wobbes T, Veth RP, Gelderman WA (1997) Operative management of impending pathological fractures: a critical analysis of therapy. J Surg Oncol 64: 190-194.

17. Zickel RE, Mouradian WH (1976) Intramedullary fixation of pathological fractures and lesions of the Subtrochanteric region of the femur. J Bone Joint Surg 58: 1061-1066.

18. Colyer RA (1986) Surgical stabilization of pathological neoplastic fractures. Curr Probl Cancer 10: 117-168. 